\title{
How to conduct research on burnout: advantages and disadvantages of a unidimensional approach in burnout research
}

\author{
V Brenninkmeijer, N VanYperen
}

Occup Environ Med 2003;60(Suppl I):i16-i20

See end of article for authors' affiliations

Correspondence to: Dr V Brenninkmeijer, TNO Work \& Employment, Team UBS2 (Social Security: Policy and Performance), PO Box 718, 2130 AS

Hoofddorp, Netherlands; v.brenninkmeijer@ arbeid.tno.nl

Accepted 12 September 2002

\begin{abstract}
When conducting research on burnout, it may be difficult to decide whether one should report results separately for each burnout dimension or whether one should combine the dimensions. Although the multidimensionality of the burnout concept is widely acknowledged, for research purposes it is sometimes convenient to regard burnout as a unidimensional construct. This article deals with the question of whether and when it may be appropriate to treat burnout as a unidimensional variable, and presents a decision rule to distinguish between people high and low in burnout. To develop a guideline for obtaining a dichotomous measure of burnout, the scores on the Utrecht Burnout Scale (UBOS) of 44 well functioning individuals were compared with the scores of 29 individuals diagnosed as suffering from burnout. Based on these data, the authors recommend the "exhaustion +1 " criterion for research in non-clinical populations. Following this criterion, individuals can be considered as burnt out when they report, compared to a norm group, high emotional exhaustion, in combination with high depersonalisation or low personal accomplishment. The criterion may be used to estimate the percentage in a sample of individuals in a state of burnout.
\end{abstract}

$\mathrm{B}$ urnout, a state of mental exhaustion resulting from chronic stress in the working situation, is a "hot" issue. In the mass media, in television programmes, magazines, and newspapers, burnout currently receives a considerable amount of attention. In scientific journals as well, the literature on burnout is abundant: in August 2002, entering the term "burnout" in psycINFO, the literature database of the American Psychological Association, resulted in 3153 hits.

Burnout is commonly regarded as a syndrome comprising three dimensions. ${ }^{12}$ The first, most central aspect is emotional exhaustion. ${ }^{3}$ One feels mentally drained and "worn out" or "empty". The second symptom, depersonalisation, refers to a cynical, negative attitude towards one's work or towards the recipients of one's service. For instance, teachers may make cynical comments about their pupils or physicians about their patients. Finally, the third symptom of burnout is reduced personal accomplishment, which denotes a reduced sense of competency in comparison to one's past functioning. The Maslach Burnout Inventory ${ }^{4}$ contains subscales for each dimension of burnout and is the commonly used inventory to assess burnout. To respect the multidimensional structure of

\section{Main messages}

- By discussing different conceptualisations of burnout, this article provides guidelines for the presentation of results obtained with the Maslach Burnout Inventory.

- It is recommended that the choice for a specific conceptualisation of burnout not only depends on one's main research interest, but also on the complexity of the design and the distributional properties of the sample.

- To obtain a dichotomous burnout score, the "exhaustion + $1^{\prime \prime}$ criterion is recommended for research in non-clinical populations.

- Following this criterion, individuals can be considered as burnt out when they report, compared to a norm group, high emotional exhaustion, in combination with high depersonalisation or low personal accomplishment.

- This criterion is useful for computing burnout percentages in samples and for investigating differences between individuals low and high in burn out. the burnout syndrome, researchers generally report results separately for each dimension.

When conducting research on burnout, it may be difficult to decide whether one should report results separately for each burnout dimension or whether one should combine the dimensions. Although it is on conceptual grounds preferable to treat burnout as a multidimensional construct, for researchers in the field of burnout it is sometimes more convenient to treat burnout as a unidimensional variable. The purpose of this article is to offer guidelines about the presentation of results for researchers in the field of burnout, in particular for those who are employing the Maslach Burnout Inventory. It deals with the question of whether and when it may be appropriate to treat burnout as a unidimensional construct, rather than reporting results separately for the underlying dimensions. Moreover, attention is paid to the pros and cons of conceptualising burnout as a dichotomous versus a continuous variable. Finally, we present an empirical study in which we developed a decision rule for research purposes that distinguishes between people high and low in burnout. Such a decision rule would be helpful in estimating the percentage of burnt out individuals in a specific sample.

\section{Multidimensionality versus unidimensionality}

According to various researchers, the multidimensional structure of the concept of burnout does not allow for combining the different components into a unidimensional variable. ${ }^{56} \mathrm{~A}$ first objection to a unidimensional approach is that the associations between the dimensions themselves and with other

\section{Policy implications}

- When conducting research in the field of burnout, or when designing interventions to combat burnout, it is important to reflect carefully on the most appropriate conceptualisation of burnout.

- The choice for a specific conceptualisation of burnout may be determined by one's main research interest, the complexity of the design, and the distributional properties of the sample.

- To compute the burnout percentage in a specific sample, the "exhaustion +1 " criterion is recommended 
variables are complex. ${ }^{57}$ Combining the dimensions would result in a considerable loss of information. A second objection is that dimensions have been constructed in such a way that they are maximally independent from one another. ${ }^{4}$ A third objection is that the role of the dimensions in the burnout process may possibly vary in the different phases of the process of burning out. ${ }^{8-10}$ On the other hand, it has been argued that burnout is strongly dominated by emotional exhaustion and that the additional weight of the other two dimensions is restricted (for example, Shirom ${ }^{11}$ ). It is a common finding that variables such as job demands are more strongly associated with emotional exhaustion than with the other two burnout dimensions. ${ }^{12}$

Although the multidimensionality of burnout is widely acknowledged, there are theoretical and practical reasons to consider burnout as a single construct. From a theoretical viewpoint, it is important to note that burnout has been proposed as a specific work related syndrome that consists of the three burnout dimensions. As with other multidimensional syndromes, the multifaceted nature of the burnout syndrome does not imply that we should abandon the overall concept of burnout. In contrast, conducting research and theorising on the overall concept of burnout may sometimes help us to advance our knowledge in a more thorough way than research on the separate, underlying dimensions. It should be noted that a unidimensional approach is not uncommon in research on related multidimensional concepts in psychology. For example, depression researchers often focus on differences among depressed versus non-depressed individuals, but ignore effects of the subdimensions of depression, such as depressed affect or the absence of positive affect. ${ }^{13}$ Moreover, with respect to the CES-D scale, a scale designed to measure depressive symptomatology in the general population, it is warned against undue emphasis on the subdimensions because of the high internal consistency of the total scale. ${ }^{14}$
With regard to the practical reasons, a unidimensional approach would have the advantage of simplifying results considerably. Reporting findings for the variable "burnout", rather than separately for the underlying dimensions, may give a better understanding of the outcomes, especially when complex effects are studied. For instance, one may examine the successfulness of two particular types of training in improving mental health, physical health, and commitment, whereby one is interested in differential effects for individuals low and high in burnout and in moderating effects of gender. The results of this complex, fictitious experiment may be more easily understood when the burnout dimensions are combined. In this way, one needs less effort to integrate the findings of the separate dimensions. To illustrate, what should one conclude when a significant effect is found for emotional exhaustion, but a marginally significant effect for depersonalisation, and a non-significant effect for reduced personal accomplishment? By combining the burnout dimensions, overall effects of burnout become more visible. And of course, one may decide to conduct additional analyses to provide detailed information about the underlying dimensions for those who are interested.

Whether the advantages of a unidimensional approach outweigh the disadvantages, may be determined by the researcher's focal interest and, somewhat relatedly, by the complexity of the research design. Figure 1 presents a decision tree for choosing between a three dimensional, continuous, or dichotomous conceptualisation of burnout. As shown in this decision tree, researchers who are primarily interested in the underlying burnout dimensions may report results for each dimension separately. This would apply, for example, to researchers focusing on interactions between the different components of burnout, such as in the study of phase models of burnout (for example, Golembiewski and colleagues ${ }^{8}$ ), or to those who are interested in the differential effects of the burnout dimensions on other variables. ${ }^{15}$ For instance,

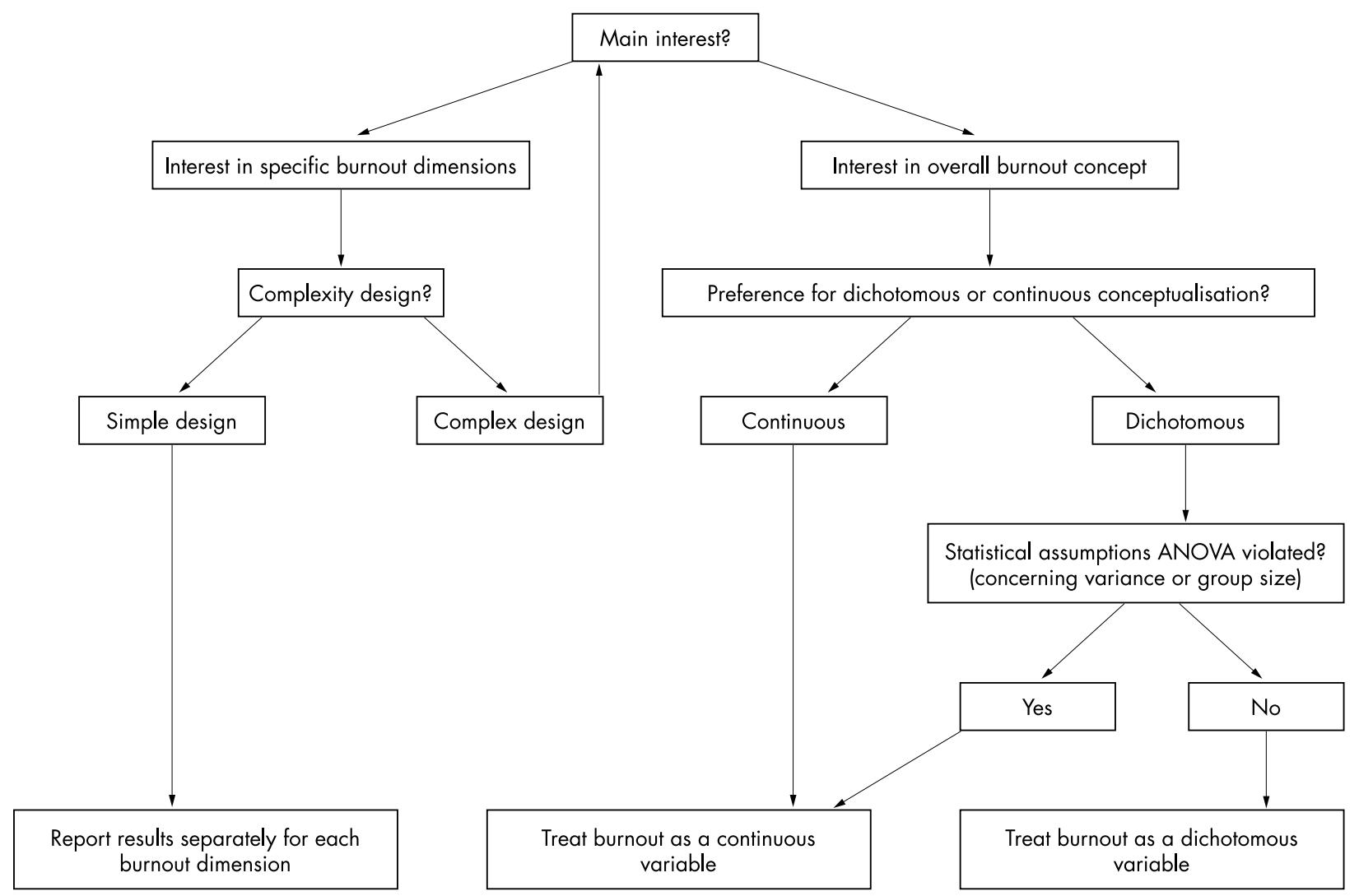

Figure 1 Decision tree for choosing between a three dimensional, continuous, or dichotomous conceptualisation of burnout. 
researchers may want to know which dimension is most predictive of turnover. They may also want to examine whether the antecedents of burnout, such as personality characteristics, work related attitudes, and work and organisational characteristics, are differentially related to the three burnout dimensions. Finally, researchers evaluating interventions to reduce burnout may wish to know precisely which burnout dimensions have (and have not) improved as a result of the intervention (for example, Cooley and Yovanoff ${ }^{16}$ ).

Researchers interested in the overall concept of burnout, rather than in the underlying dimensions, may decide to combine the burnout dimensions into a single score. This may be useful, for example, for researchers who wish to estimate the prevalence of burnout in a sample (for example, Schaufeli and Enzmann ${ }^{12}$ ). In addition, researchers may sometimes want to examine differences between "healthy" individuals and individuals in a state of burnout. In particular, this may apply to researchers working with complicated research designs. For reasons of parsimony, a complex design (for example, a design with three interacting factors and several dependent variables) may urge researchers to focus on the overall concept of burnout and to pay less attention to the underlying dimensions.

All in all, the specific research question, as well as the complexity of the research question, may be taken into consideration when choosing between a multi- or unidimensional approach. A unidimensional approach implies a substantial loss of information, though it may increase the clarity of the presented results.

\section{Dichotomous versus continuous conceptualisation}

Once opted for a unidimensional approach, it is important to decide between a dichotomous or continuous conceptualisation of burnout (see fig 1). Although this issue is applicable to almost any psychological variable, including the separate burnout dimensions, and although it is widely covered in the methodological literature, we believe that it may be useful to discuss it briefly with respect to the measurement of burnout. For computing burnout percentages, it is obvious that burnout should be treated as a dichotomous variable. However, for studying effects of burnout, or differences between individuals low and high in burnout, the answer is more complicated. Clearly, the researcher's ideas about the distribution of burnout, in general or in a specific sample, come into play: is burnout found in different gradations, or is it rather a matter of "all or nothing"? Moreover, methodological issues may influence the choice for a dichotomous or a continuous variable. Opting for burnout as a dichotomous independent variable permits the use of $t$ tests, ANOVAs, or MANOVAs ( that is, univariate or multivariate analyses of variance). Nevertheless, it is sometimes difficult to meet the criteria for these statistical analyses. An important precondition is homogeneity of variance; in particular, the largest variance should not be associated with the smallest group. ${ }^{17}$ Moreover, small group sizes generally reduce the power of these tests. In burnout research, it is often the case that the group with burnout is small and that the variance in this group is large. In this situation, it may be wiser to conduct regression analyses with burnout as a continuous variable. Although regression analysis also assumes constant variance, the use of continuous variables eliminates the problem of small group size. Nonetheless, it should be noted that heterogeneity of variance can itself be the subject of investigation. ${ }^{18}$ For example, it may be informative to know that individuals suffering from burnout vary largely in their emotional reaction to certain threats.

\section{How to combine the burnout dimensions into a dichotomous score}

There are several ways to combine the burnout dimensions into a unidimensional score. According to Golembiewski and colleagues, ${ }^{8}$ the three components of burnout can be combined into eight phases, or eight gradations. Individuals would be in the severest state of burnout when they score on all three dimensions above a "universal norm". This universal norm is the median in a norm group consisting of employees from a large federal agency. However, Schaufeli and Enzmann ${ }^{12}$ view the criteria used by Golembiewski et al as quite liberal and arbitrary, and plead for the development of clinically validated, local criteria.

We conducted a study to provide a decision rule for computing a dichotomous burnout score by comparing of group of "healthy individuals" with a group of individuals who were clinically diagnosed as burnt out. ${ }^{19}$ The purpose of this study was to yield a decision rule with large distinguishing power for individuals in a non-clinical context. This decision rule would not be meant as a tool for individual diagnostics, but as an instrument for research purposes-that is, for conclusions about non-clinical samples. Therefore, we investigated how the scores on the three dimensions of the Utrecht Burnout Scale (UBOS) ${ }^{20}$ could be validly combined into a qualification "high" or "low" in burnout, whereby we primarily aimed to keep the chance of a type 1 error (that is, a false positive: someone low in burnout is falsely qualified as high in burnout) around the conventional level of 5\%. The qualification "high in burnout" signifies that individuals are in a state of burnout or are at a very high risk of getting into a state of burnout. Thus, these individuals would qualify for a therapeutic treatment to combat or prevent burnout.

We not only examined the predictive power of the UBOS dimensions by means of logistic regression, we also looked for a practically useful categorisation based on the norm scores of the UBOS, ${ }^{20}$ a categorisation that has the advantage of being independent of the current sample. Because emotional exhaustion is considered to be the core symptom of burnout, we examined only categorisations in which high emotional exhaustion was a precondition for the qualification burnout. The decisive weight of the two remaining symptoms was varied.

\section{METHODS}

\section{Procedure and participants}

The data of the study originate from a study by Wagenvoort and colleagues, ${ }^{21}$ in which 73 individuals participated. Analogous to the study by Wagenvoort et al, we employed both a clinical group and a control group. The clinical group consisted of 29 participants ( 25 men and four women) who were suffering from burnout and who were selected via an institute for work related problems and psychotherapy. The mean age in this group was 45 years (SD 6.46). Among clients who were treated for burnout (see below for the criteria of burnout), the therapist controlled with a checklist to what extent the clinical picture corresponded to the criteria of the diagnosis neurasthenia. Only clients who met the diagnostic criteria were requested by their therapist to fill in a questionnaire and to return the filled in questionnaire.

The control group consisted of 44 individuals (26 men and 18 women) who were not treated for burnout and who were working in various professions. The average age in this group was 43 years ( SD 9.89). Individuals were asked if they wanted to participate in a study about the relation between personality characteristics and the way people cope with problems. The questionnaire could be filled in at home and could subsequently be returned. The response percentage in this group was $95 \%$.

In the study by Wagenvoort and colleagues, ${ }^{21}$ the clinical group appeared to diverge from the non-clinical group in several respects. For instance, the clinical group scored higher on exhaustion and distance, and lower on competence. In addition, individuals in the clinical group were lower in self esteem, tended to somatise more, and were more neurotic. 
Moreover, individuals in this group were shy and not very extraverted in comparison to the non-clinical group.

We used logistic regression to examine how well the UBOS dimensions predicted burnout. In addition, we investigated three possible categorisations that were based on the UBOS norm scores. In these categorisations, individuals were classified as high in burnout when they were characterised by:

- High exhaustion

- High exhaustion, accompanied by high distance or low competence

- High exhaustion, accompanied by high distance and low competence.

"High" means "scoring in the 75th percentile or higher", whereas "low" refers to "scoring in the 25 th percentile or lower". ${ }^{20}$

\section{Measures}

\section{Burnout diagnosis}

The diagnosis burnout was made when the patient met the criteria for the diagnosis of neurasthenia following the ICD-10 classification (International Classification of Diseases of the World Health Organisation ${ }^{22}$ ), and when the clinical picture resulted from a long lasting process of relative overload. The diagnostic criteria of neurasthenia following the ICD-10 are as follows: persistent and distressing complaints of either increased fatigue after mental effort, or bodily weakness and exhaustion after minimal effort. Furthermore, the patient experiences at least two of the following complaints: muscular aches and pains, dizziness, tension headaches, sleeping disturbance, inability to relax, irritability, and dyspepsia (stomach and intestinal complaints). Moreover, the clinical picture may not correspond to a more specific ICD-10 disorder (for example, an anxiety or depressive disorder). When the complaints were work related and when professional qualities had declined, this was interpreted as additional support for the diagnosis of burnout. ${ }^{23}$

\section{Burnout questionnaire}

Burnout was assessed with the Utrecht Burnout Scale (UBOS), which consists of 16 items. ${ }^{20}$ This scale has been developed to also measure burnout outside the human services and consists of the following three subscales: exhaustion ( $\mathrm{U}$ scale), distance (D scale), and competence (C scale). The three factor structure fits reasonably well to empirical findings. The internal consistency is good and the stability is reasonably good. Items could be responded to on a seven point scale varying from 0 ("never") to 6 ("always, daily").

\section{RESULTS}

To determine the predictive value of the UBOS dimensions, we performed logistic regression analyses with burnout (coded as 0 for "burnout" and 1 for "no burnout") as dependent variable. The model, consisting of the three UBOS dimensions, had more predictive power for burnout compared to the constant (model $\chi^{2}(3)=65.26, \mathrm{p}<0.001$ ) and yielded a burnout percentage of $9.1 \%$ in the non-clinical group and $86.2 \%$ in the clinical group. Hence, the chance of a type 1 error was $9.1 \%$. However, only exhaustion appeared to be a significant predictor $(\mathrm{B}=-2.10, \mathrm{SD} 0.54, \mathrm{p}<0.001)$; distance $(\mathrm{B}=0.07$, SD $0.51, \mathrm{NS})$ and competence $(\mathrm{B}=0.51, \mathrm{SD} 0.55$, NS) were not significant predictors, which may be attributed to the high intercorrelations between the three dimensions $\left(r_{\text {ee-d }}=0.67\right.$, $\left.\mathrm{r}_{\text {ee-c }}=-0.46, \mathrm{r}_{\mathrm{c}-\mathrm{d}}=-0.34, \mathrm{p}<0.01\right)$. To determine the optimal critical score for exhaustion, we performed a logistic regression with only exhaustion as a predictor. This analysis resulted in the same burnout percentages as the first analysis (see table 1). This analysis yielded also the following formula: burnout $=6.16-2.12 \times$ exhaustion. Filling in the critical value of burnout $(0.5)$, resulted in a critical score of 2.67 for
Table 1 Definition of burnout and resulting burnout percentage

\begin{tabular}{lcl}
\hline & \multicolumn{2}{l}{ Burnout \% } \\
\cline { 2 - 3 } Definition of burnout & Control group & Clinical group \\
\hline EE $\geqslant 2.67^{*}$ & 9.1 & 86.2 \\
EE high & 15.9 & 91.3 \\
EE high, and D high or C low & 6.8 & 69.0 \\
EE high, D high, and C low & 0.0 & 41.4
\end{tabular}

^EE, emotional exhaustion; D, distance, C, competence.

exhaustion; following this categorisation, persons with a score similar or higher than 2.67 were categorised as burnt out.

In addition to the logistic regression analyses, the accuracy of three categorisations based on the UBOS norm scores were examined. ${ }^{20}$ The categorisation in which high exhaustion, and high distance or low competence were conditions for burnout ("exhaustion +1 ") seemed to be the most effective categorisation to distinguish between individuals high and low in burnout. This resulted in a fairly small chance $(6.8 \%)$ of an inaccurate qualification of burnout (that is, a false positive), although the burnout percentage in the clinical group $(69.0 \%)$ was somewhat conservative (see table 1). When high exhaustion, high distance, and low competence were all three conditions for burnout, this reduced the chance of a false positive to zero, but the burnout percentage in the clinical group was very low $(41.4 \%)$. A categorisation in which only high exhaustion was a condition for the qualification burnout yielded a burnout percentage of $15.9 \%$ in the control group and $93.1 \%$ in the clinical group.

\section{DISCUSSION}

When conducting research on burnout, it may be difficult to decide whether one should report results separately for each burnout dimension or whether one should combine the dimensions. In research on burnout, results are often presented for each dimension separately. This approach respects the multidimensionality of the concept of burnout and is the appropriate procedure for researchers interested in the underlying dimensions of burnout. However, when one is primarily interested in differences between individuals high and low in burnout, it seems more convenient to combine the dimensions. This improves the understandability and clarifies the results, especially when complex research questions, such as three way interactions, are studied. Moreover, from a theoretical viewpoint, it is important to note that researchers have proposed burnout as a specific syndrome, thereby underlining the importance of an overall conceptualisation of burnout. In fig 1 , a decision tree is depicted for choosing between a three dimensional, continuous, or dichotomous conceptualisation of burnout.

We conducted an empirical study to develop a decision rule for research purposes that distinguishes between people high and low in burnout. This criterion would be useful for computing burnout percentages in samples and for investigating differences between individuals low and high in burnout. We found that a categorisation in which both high exhaustion and high distance or low competence were conditions for burnout ("exhaustion +1 "), resulted in a fairly small chance $(6.8 \%)$ of an inaccurate qualification of burnout (that is, a false positive). The chance of an accurate qualification of burnout with this decision rule was $69.0 \%$ (see table 1 ). The advantage of this categorisation is that it is based on existing norm scores that are independent of the current sample. However, logistic regression revealed that an alternative categorisation, in which we applied a critical value of 2.67 on emotional exhaustion, was on average most accurate. Although this decision rule resulted in a larger type 1 error 
(9.1\%), the chance of an accurate diagnosis of burnout was considerably larger $(86.2 \%)$. The drawback of this decision rule is that it is based only on a small sample.

Recently, the "exhaustion +1 " rule is referred to in the manual of the Utrecht Burnout Scale. ${ }^{24}$ In addition, in a study by Brenninkmeijer and colleagues, ${ }^{25}$ the "exhaustion +1 " criterion has been successfully applied to examine differences between individuals low and high in burnout. The purpose of that study was to examine whether teachers high and low in burnout differ in the perception of being superior to others. Participants were asked to generate information about inferior and superior others. Perceived superiority was assessed by response latencies and the quality of the information generated. As expected, among those high in burnout (following the "exhaustion +1 " criterion), positive superiority (that is, feeling better than others) was reduced, whereas the perception of negative superiority (that is, feeling less bad than others) was intact. Thus, the "exhaustion +1 " rule seems to be an effective categorisation for mapping differences between individuals high and low in burnout.

It should be noted that the results in this study were obtained in a relatively small sample and with a preliminary version of the UBOS. In 2000 a final version of the UBOS was published, with slightly different items and revised norm scores. ${ }^{24}$ Future research, preferably in a larger sample, would be necessary to examine the accuracy of the developed criterion for researchers using the revised version of the UBOS. Nevertheless, the current criterion is formulated in terms of "high" scores (75th percentile or higher) on emotional exhaustion and distance and "low" scores (25th percentile or lower) on competence, and may therefore be useful for other burnout inventories as well, including the new version of the UBOS.

When the group with burnout is too small or too heterogeneous for reliable analyses, which may often be the case, it seems better to employ a continuous burnout scale. Little is known, however, about the way in which the dimensions can be most accurately combined into a continuous score. Our empirical study ${ }^{19}$ would suggest that one might use emotional exhaustion as a sole, continuous predictor, because this was the only dimension that reached significance in the logistic regression analysis. We feel, however, that abandoning the other two burnout dimensions may be a somewhat drastic conclusion from our study, especially as our sample was not very large. Moreover, a few studies have presented their results with a combined, continuous measure of burnout with high internal consistency. In a study by Buunk and colleagues, ${ }^{26}$ all items of the Maslach Burnout Inventory were summed up, which resulted in a Cronbach's alpha of 0.87. In two other studies, this procedure resulted in a highly consistent burnout scale (Cronbach's alphas $>0.80) \cdot{ }^{27}$ Hopefully, future research will teach us about the preferable procedure for creating a single, continuous burnout variable.

\section{Authors' affiliations \\ V Brenninkmeijer, N VanYperen, University of Groningen, \\ Netherlands}

This study was conducted as part of the Netherlands concerted research action on "Fatigue at Work" granted by the Netherlands Organisation of Scientific Research (NWO: grant no. 580-02.230)

\section{REFERENCES}

1 Maslach C, Jackson SE. The measurement of experienced burnout. J Occup Behav 1981;2:99-113.
2 Maslach C, Schaufeli WB, Leiter MP. Job burnout. Annu Rev Psychol 2000;52:397-422

3 Schaufeli WB, Maslach C, Marek T, eds. Professional burnout: recent developments in theory and research. London: Taylor and Francis, 1993.

4 Maslach C, Jackson SE. The Maslach Burnout Inventory. Manual edition. Palo Alto, CA: Consulting Psychologists Press, 1986.

5 Maslach C. Burnout: a multidimensional perspective. In: Schaufeli WB Maslach C, Marek T, eds. Professional burnout: recent developments in theory and research. Washington, DC: Taylor and Francis, 1993:19-32.

6 Schaufeli WB, Van Dierendonck D. Burnout, een concept gemeten: De Nederlandse versie van de Maslach Burnout Inventory (MBI-NL) [Burnout, the measurement of a concept: the Dutch version of the Maslach Burnout Inventory (MBI-NL)]. Gedrag en Gezondheid 1994;22:153-72.

7 Koeske GF, Koeske RD. Construct validity of the Maslach Burnout Inventory: a critical review and reconceptualization. J Appl Behav Sci 1989:25:131-44.

8 Golembiewski RT, Boudreau RA, Munzenrider RF, et al. Global burnout: a world-wide pandemic explored by the phase model. Monographs in organizational behavior and industrial relations, Vol. 21 Greenwich, CT: JAl Press, 1996.

9 Leiter MP. Burnout as a developmental process: consideration of models. In: Schaufeli WB, Maslach C, Marek T, eds. Professional burnout: recent developments in theory and research. Washington, DC: Taylor and Francis, 1993:237-50.

10 Van Dierendonck D, Schaufeli WB, Buunk BP. Toward a process mode of burnout: results from a secondary analysis. European Journal of Work and Organizational Psychology 2001;10:41-52.

11 Shirom A. Burnout in work organizations. In: Cooper CL, Robertson I, eds. International review of industrial and organizational psychology. New York: Wiley \& Sons, 1989:25-48.

12 Schaufeli WB, Enzmann D. The burnout companion to study and practice: a critical analysis. London: Taylor \& Francis, 1998.

13 Ahrens AH, Alloy LB. Social comparison processes in depression. In: Buunk BP, Gibbons FX, eds. Health, coping, and well-being: perspectives from social comparison theory. Hillsdale, NJ: Erlbaum, 1997:389-410.

14 Radloff LS. The CES-D scale: a self-report depression scale for research in the general population. Applied Psychological Measurement 1977; 1:385-401.

15 Lee RT, Ashforth BE. A meta-analytic examination of the correlates of the three burnout dimensions of job burnout. J Appl Psychol 1996;81:123-33.

16 Cooley E, Yovanoff P. Supporting professionals at risk: evaluating interventions to reduce burnout and improve retention of special educators. Exceptional Children 1996;62:336-55.

17 Stevens J. Applied multivariate statistics for the social sciences, 2 nd edn. Hillsdale, NJ: Lawrence Erlbaum Associates, 1992.

18 Tacq J. Multivariate analysis techniques in social science research. London: Sage Publications, 1997

19 Brenninkmeijer V, VanYperen NW. Diagnostiek van burnout door middel van de Utrechtse Burnout Schaal [Diagnosing burnout by means of the Utrecht Burnout Scale]. Nederlands Tijdschrift voor de Psychologie 1999;54:105-8.

20 Schaufeli WB. Utrechtse Burnout Schaal (UBOS): Voorlopige handleiding [Utrecht Burnout Scale (UBOS): Preliminary manual]. Internal report, section Psychology of Work, Health and Organization, University of Utrecht, Netherlands, 1995

21 Wagenvoort MA, VanYperen NW, Hoogduin CAL, et al. Persoonskenmerken en burnout: De mediërende rol van copingstijl [Personality characteristics and burnout: The mediator role of coping style]. Nederlands Tijdschrift voor de Psychologie 1998;53:128-43.

22 WHO. The ICD-10 classification of mental and behavioural diseases: clinical description and diagnostic guidelines. Geneva: WHO, 1992.

23 Hoogduin CAL, Schaap CPDR, Methorst GJ. Burnout: Klinisch beeld en diagnostiek. [Burnout: clinical picture and diagnostics]. In: CAL Hoogduin, CPDR Schaap, Kladler AJ, Hoogduin WA, eds. Behandelingsstrategieën bij burnout [Treatment strategies of burnout]. Houten/Diegem, Netherlands: Bohn Stafleu Van Logum, 1996:31-41.

24 Schaufeli WB, Van Dierendonck D. UBOS: Utrechtse Burnout Schaal. Handleiding [UBOS: Utrecht Burnout Scale. Manual]. Lisse, Netherlands: Swets and Zeitlinger, 2000.

25 Brenninkmeijer V, VanYperen NW, Buunk BP. I am not a better teacher, but others are doing worse: burnout and perceptions of superiority among teachers. Social Psychology of Education 2001;4:259-74.

26 Buunk BP, Ybema JF, Gibbons FX, et al. The affective consequences of social comparison as related to professional burnout and social comparison orientation. European Journal of Social Psychology 2001;31:337-51.

27 Brenninkmeijer V, VanYperen NW, Buunk BP. Burnout, social comparison orientation and the affective consequences of social comparison information. Manuscript submitted for publication, 2000 